\title{
Multi-target vital signs detection using frequency-modulated continuous wave radar
}

Yong Wang, Yuzhu Shui, Xiaobo Yang* ${ }^{*}$, Zhaoyu Li and Wen Wang

*Correspondence:

yangxb@cqupt.edu.cn

School of Communication

and Information

Engineering, Chongqing

University of Posts

and Telecommunications,

Chongqing, China

\begin{abstract}
Respiration and heartbeats rates are important physiological assessment indicators that provide valid prior-knowledge for the diagnosis of numerous diseases. However, most of the current research focuses on the vital signs measurement of single target, and multi-target vital signs detection has not received much attention. In this paper, we use frequency-modulated continuous wave (FMCW) radar to measure the vital signs signals of multi-target. First, we apply the three-dimensional fast Fourier transform (3D-FFT) method to separate multiple targets and get their distance and azimuth information. Subsequently, the linear constrained minimum variance-based adaptive beamforming (LCMV-ADBF) technique is proposed to form a spatially distributed beams on the targets of interest directions. Finally, a compressive sensing based on orthogonal matching pursuit (CS-OMP) method and rigrsure adaptive soft threshold noise reduction based on discrete wavelet transform (RA-DWT) method are present to extract the respiratory and heartbeat signals. We perform tests in a real experimental environment and compare the proposed method with reference devices. The results show that the degrees of agreement for respiratory and heartbeat are $89 \%$ and $87 \%$, respectively, for two human targets. The level of agreement for respiratory and heartbeat is $87 \%$ and $85 \%$, respectively, for three human targets, proving the effectiveness of the proposed method.
\end{abstract}

Keywords: Vital signs detection, FMCW, 3D-FFT, LCMV-ADBF

\section{Introduction}

Respiratory and heartbeat signals are important biosignals, which not only provide the physical health status of patients, but also facilitates the daily management of some public health systems [1]. Moreover, research shows that both respiration and heart rate are essential forecasters of sudden illnesses such as cardiac arrest [2]. Currently, the respiratory and heartbeat detection approaches are conventional contacted (ECG, optics, airflow sensing, chest wall mechanical displacement sensing and blood pressure sensing) and radar-based non-contact detection [3-10]. Although the contact vital signs detection has a high accuracy and stability [11]. However, it is not friendly for patients with burns and skin infections, etc. In contrast, the radar sensors are used to extract respiratory and heartbeat signals using various signal processing methods by detecting millimeter-scale displacement of the thoracic surface. author(s) and the source, provide a link to the Creative Commons licence, and indicate if changes were made. The images or other third party material in this article are included in the article's Creative Commons licence, unless indicated otherwise in a credit line to the material. If material is not included in the article's Creative Commons licence and your intended use is not permitted by statutory regulation or exceeds the permitted use, you will need to obtain permission directly from the copyright holder. To view a copy of this licence, visit http:// creativecommons.org/licenses/by/4.0/. 
This makes the radar detection system providing great potential and promise for development.

Up to now, most of the efforts have focused on the study of single target with known location. However, there is an urgent need for emergency rescue [12], ward monitoring $[13,14]$, and many other scenarios that require simultaneous monitoring of multiple targets. The frequency-modulated continuous wave (FMCW) radar can detect both absolute and relative displacements of the human thoracic surface, and it has greater advantages especially in the field of multi-target vital signs signals detection [15]. Various solutions have been developed using FMCW radar, in which the distance difference and spatially distributed beams of multiple targets are widely used [16-25]. Ref. [16] uses advanced signal processing method with adaptive boundaries to improve the distance resolution and achieve short-range multi-target vital sign detection. A mixed radar system combining FMCW mode and interferometry mode is proposed in [17]. Specifically, the FMCW mode is responsible for range detection and interferometry mode is responsible for vital activity (respiration, heartbeat and body movement) detection. Lee et al. [18] also apply a 24 GHz FMCW radar for heart rate estimation. They use the phase change information caused by distance and Doppler to eliminate the noise in the environment and reduce the interference between multiple targets. The separation of multi-target vital signs at a minimum distance interval of $40 \mathrm{~cm}$ is finally achieved. However, the human targets in these cases are often at different distance ranges, without considering the pattern of different targets at the same distance. The authors in [19-21] combine the multiple signal classification (Music) and rotation invariant technique to improve the resolution of DOA estimation by using the orthogonality of signal and noise subspaces. However, the resolution performance of these methods is largely affected by the number of targets, signal-to-noise ratio (SNR) and data length, making the stability and efficiency of the system a great challenge. In [22, 23, 25], a mechanical scanning (MS) method is proposed to achieve beam scanning by rotating the radar antenna as a way to achieve multi-target detection. However, the simultaneous detection of multiple targets is impossible due to the limitation of turntable speed. Fang et al. [24] design a radio frequency (RF) conversion radar system by adding RF switches as multi-plexers to achieve simultaneous detection of two targets' vital signals from different distances of the antenna within the resolution limitation. However, the authors continue to ignore the case where different targets are at the same distance. Moreover, the authors do not extend the two to multiple targets.

In this paper, we propose to use the FMCW radar to achieve multi-target vital signs detection system. The main contributions of this paper are as follows.

Firstly, the echo signal of the FMCW radar array antenna is modeled, and the principle of the FMCW radar is described. Through the theoretical derivation of the virtual antenna expansion technology, the multi-target detection expression form of the intermediate frequency (IF) signal is completely given, and the echo modeling of the vital sign is successfully realized.

Secondly, we propose to apply the three-dimensional fast Fourier transform (3DFFT) method to estimate the number and angle of the target automatically. Then, the ADBF method based on linearly constrained minimum variance (LCMV) is adopted 
to weaken the interference between targets and enhance the target signal. Finally, the constant false alarm rate (CFAR) method is used to successfully separate different human targets.

Finally, we build up the multi-target vital signs detection system and carry out a series of experiments to verify the effectiveness and feasibility of the CS-OMP and RA-DWT methods. The measured results show that the level of agreement for respiration and heartbeat obtained by two methods and connect devices (respiratory sensor and Apple Watch6) are more than $89 \%$ and $87 \%$, respectively, for two human targets. Moreover, the level of agreement for respiration and heartbeat are $87 \%$ and $85 \%$, respectively, for three human targets,

The remainder of the paper is organized as follows. Section II introduces the modeling of the FMCW radar-based vital signs. Section III describes the principle of multiple-input-multiple-output (MIMO) radar. The fundamentals of multi-target extraction based on 3D-FFT and LCMV-ADBF methods are introduced. Section IV introduces the extraction method of respiratory and heartbeat signals based on the CS-OMP and RADWT methods. Section V presents the experimental results of multi-target vital sign extraction, followed by concluding remarks drawn in Section VI.

\section{IF signal model of FMCW radar}

The multi-target vital signs detection system based on FMCW radar mainly extracts the phase information of respiration and heartbeat in the IF signal. The IF signal is obtained by mixing the transmitted signal and the echo signal, and then filtering out the high frequency components.

The transmit signal of FMCW radar can be expressed as

$$
s_{T}(t)=A_{T X} \cos \left[2 \pi f_{\mathrm{c}} t+\pi \frac{B}{T_{\mathrm{c}}} t^{2}+n(t)\right],
$$

where $A_{T X}$ is the amplitude of the transmitted signal, $f_{\mathrm{c}}$ is the initial frequency of the transmitted signal, $B$ is the bandwidth, $T_{\mathrm{c}}$ is the chirp pulse width, and $n(t)$ is the phase noise. Assuming that the distance between the human target and the radar is $r_{0}$, and the human chest is accompanied by a small amplitude of vibration with time. Therefore, the distance between the human chest and the radar is

$$
R(t)=r_{0}+x(t)
$$

where $x(t)=A_{\mathrm{r}} \sin \left(2 \pi f_{\mathrm{r}} t\right)+A_{\mathrm{h}} \sin \left(2 \pi f_{\mathrm{h}} t\right)$ is an expression for the human chest vibration, where $A_{\mathrm{r}}$ and $A_{\mathrm{h}}$ are the amplitudes of respiration and heartbeat, respectively. $f_{\mathrm{r}}$ and $f_{\mathrm{h}}$ are the frequencies of respiration and heartbeat, respectively. The received signal is

$$
s_{R}(t)=A_{R X} \cos \left[2 \pi f_{\mathrm{c}}\left(t-t_{\mathrm{r}}\right)+\pi \frac{B}{T_{\mathrm{c}}}\left(t-t_{\mathrm{r}}\right)^{2}+n\left(t-t_{\mathrm{r}}\right)\right],
$$

where $t_{\mathrm{r}}=\frac{2 R(t)}{c}$ is the time delay of the received signal. The received signal and the transmitted signal are mixed and then passed through a low-pass filter to obtain the IF signal. Then, the IF signal is expressed as 


$$
\begin{aligned}
S_{\mathrm{IF}} & \approx A_{\mathrm{IF}} \exp \left\{j(\underbrace{4 \pi \frac{B r_{0}}{c T_{\mathrm{c}}}}_{f_{b}} t+\underbrace{\frac{4 \pi\left[x(t)+r_{0}\right]}{\lambda}}_{\psi(t)})\right\} \\
& =A_{\mathrm{IF}} \exp \left[j\left(2 \pi f_{b} t+\psi(t)\right)\right],
\end{aligned}
$$

where $A_{\mathrm{IF}}$ is the amplitude of the IF signal, $c$ is the speed of light, $\lambda$ is the signal wavelength, $f_{b}$ is the frequency of the IF signal, and $\psi(t)$ is the phase of the IF signal, which changes with the displacement of the human chest. In order to observe the change of the human chest displacement, it is necessary to sequentially transmit multiple chirps to obtain the phase change information and ensure that the phase change occurs between two chirps or between two frames, which is equivalent to sampling. After analog-to-digital converter (ADC) sampling, the IF signal is expressed as

$$
S_{\mathrm{IF}}=A_{\mathrm{IF}} \exp \left\{j(\underbrace{2 \pi \frac{2 B r_{0}}{c T_{\mathrm{c}}} n T_{n}}_{f_{b}}+\underbrace{\frac{4 \pi\left[x\left(m T_{\mathrm{c}}\right)+r_{0}\right]}{\lambda}}_{\psi_{m}})\right\},
$$

where $m$ is the number of chirps, $n$ is the number of sampling points for each chirp, and $T_{n}$ is the sampling period.

\section{Proposed multi-target separation scheme}

The structure of the proposed method is shown in Fig. 1. It consists of three steps: angle estimation, target separation and signal extraction. After performing analog-to-digital conversion on the IF signal, we measure the range and angle of multiple targets in the radar field of view. The range-angle map is constructed according to the distance and angle

Step1. Angle Estimation

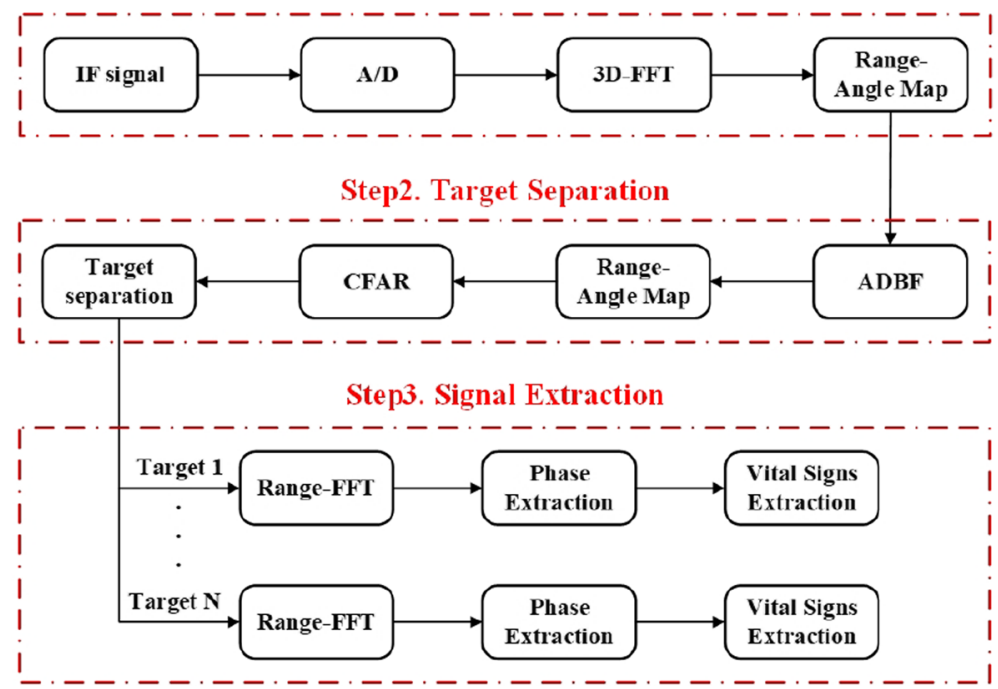

Fig. 1 Multi-human targets vital signal detection 
information. Then, the interference between multiple targets is weakened and the target signal is enhanced by ADBF technology. Afterward, multiple human target signals are separated on RAM by CFAR method. Finally, the separated individuals are detected for vital signals.

In order to show the multi-target signal processing theory of the array antennas, the signal model of the array antenna is shown in Fig. 2. The radar platform uses a two transmit and four receive antennas which can be virtually expanded into eight receiving antennas (RX5 RX8 are the expanded antennas), effectively improving the angle estimation accuracy.

\subsection{Virtual antenna extension technology}

Assuming that the distance between two adjacent receiving antennas is $d$, and the reflected signal arrival angle is $\theta$, the phase difference between different antennas is

$$
\Delta \phi=\frac{2 \pi d \sin \theta}{\lambda}
$$

Therefore, the expression form of the $i$-th antenna receiving system is

$$
S_{\mathrm{IF}}=A_{\mathrm{IF}} \exp \left\{j\left[2 \pi f_{b} n T_{n}+\psi_{m}+\frac{2 \pi}{\lambda} d_{i} \sin \theta\right]\right\} .
$$

If there are multiple targets in the radar's field of view, the received signal is cumulative. The $L$ targets at $\left(R_{l}, \theta_{l}\right)$ are expressed as

$$
S_{\mathrm{IF}}=\sum_{l=1}^{L} A_{\mathrm{IF}} \exp \left\{j\left[2 \pi f_{b_{l}} n T_{n}+\psi_{m_{l}}+\frac{2 \pi}{\lambda} d_{i} \sin \theta_{l}\right]\right\} .
$$

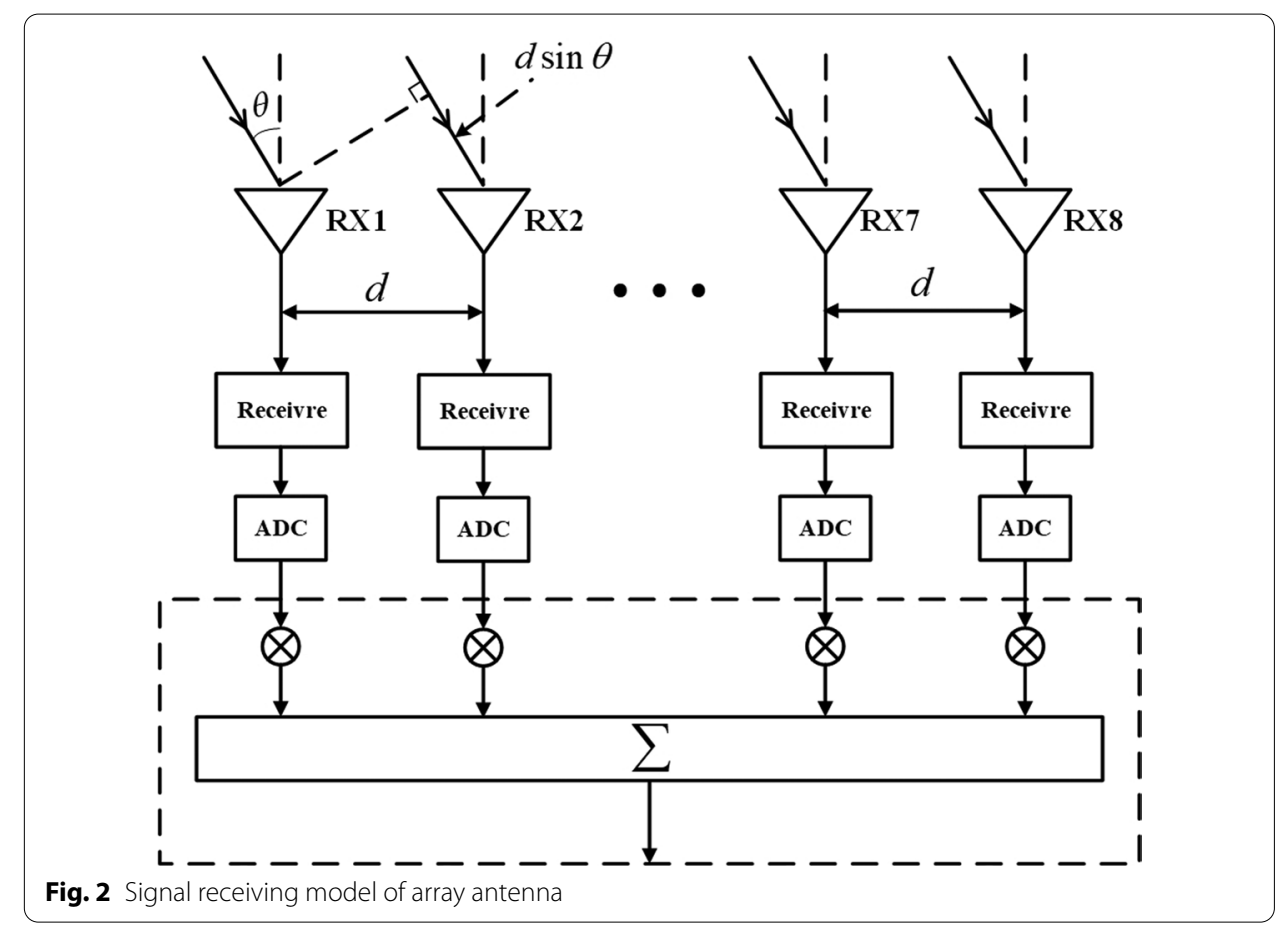


Based on the above equation, the phase change of the $i$-th receiving antenna is

$$
\psi_{m_{l}}=\frac{4 \pi R_{l}\left(m T_{\mathrm{c}}+n T_{n}\right)}{\lambda}+\frac{2 \pi}{\lambda} d_{i} \sin \theta_{l}
$$

When the targets are at the same distance unit $R_{l, o}$, the above formula will become

$$
\begin{aligned}
\psi_{m_{l}} & =\frac{4 \pi R_{l, o}}{\lambda}+\frac{4 \pi R_{l}\left(m T_{\mathrm{c}}\right)}{\lambda}+\frac{2 \pi}{\lambda} d_{i} \sin \theta_{l} \\
& =\psi_{c, i}\left(R_{l, o}, \theta_{l}\right)+\frac{4 \pi R_{l}\left(m T_{\mathrm{c}}\right)}{\lambda}
\end{aligned}
$$

where $\psi_{c, i}\left(R_{l, o}, \theta_{l}\right)$ is a constant term in the same distance-angle unit and it is not considered. The relative displacement $4 \pi R_{l}\left(m T_{\mathrm{c}}\right) / \lambda$ caused by respiration and heartbeat should be detected in the vital signs measurement.

MIMO radar multiplexing technology is the core of realizing the expansion of virtual array antennas. In this paper, the time-division multiplexing (TDM) mode is used, where TX1 and TX2 alternately transmit FM pulses using time slot interval. As shown in Fig. 3, in one chirp cycle, the 8-way virtual expansion receive antenna array receives the echo signal $S_{1}$ or $S_{2}$, respectively, to realize the virtual array expansion.

Figure 4 shows the beam direction obtained using the 8-way virtual antenna expansion technique for different numbers of receiving array elements, where $\mathrm{N}$ is the number of receive arrays. It can be seen that the beam direction map of the 8-way antenna array after virtual expansion has a narrower beam width, which improves the angular estimation resolution.

\subsection{D-FFT-based distance-angle map construction}

Limited by the distance resolution, it is difficult to distinguish two targets at almost the same distance through Range-FFT. Thus, it is necessary to construct a range-angle map (RAM) to separate the multiple human targets. The echo reflected by the target at

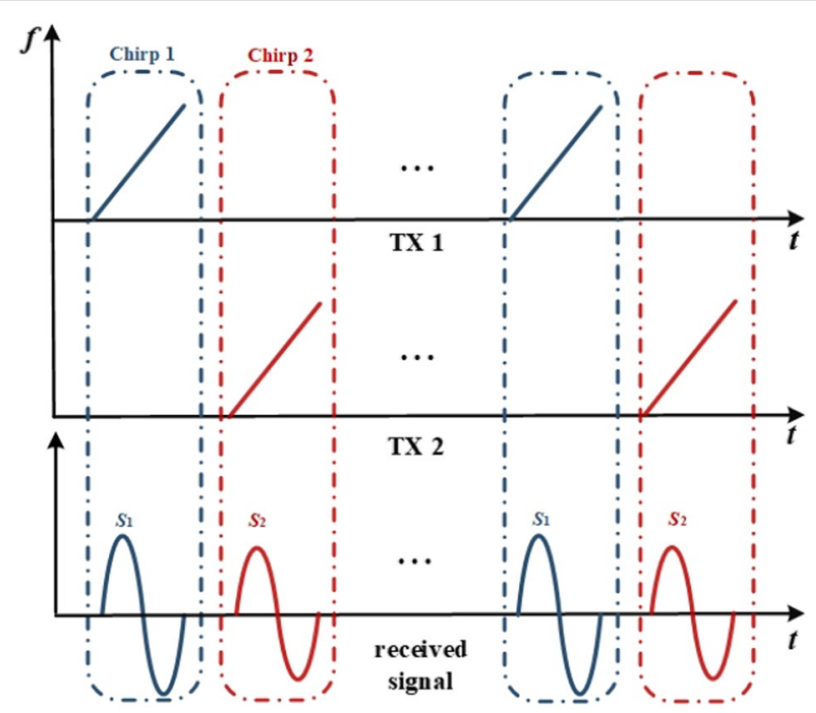

Fig. 3 TDM 


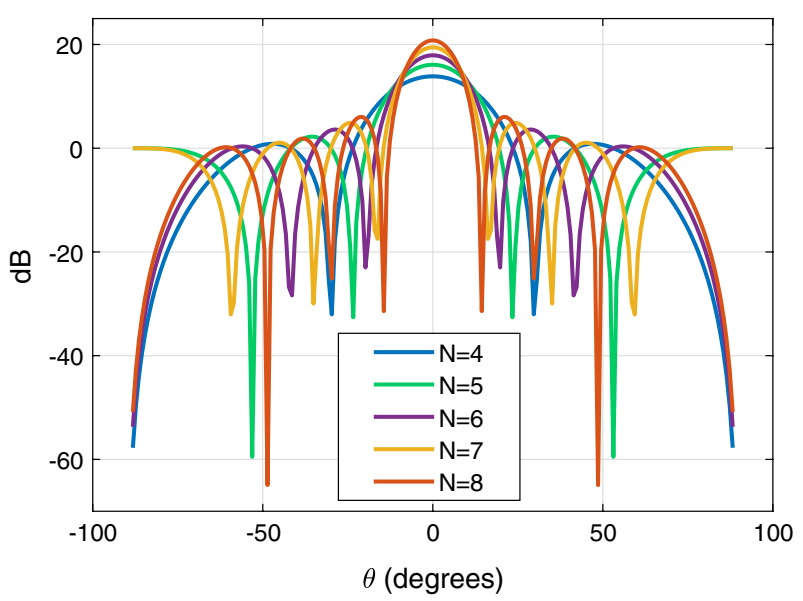

Fig. 4 Beam patterns corresponding to different receiving array elements

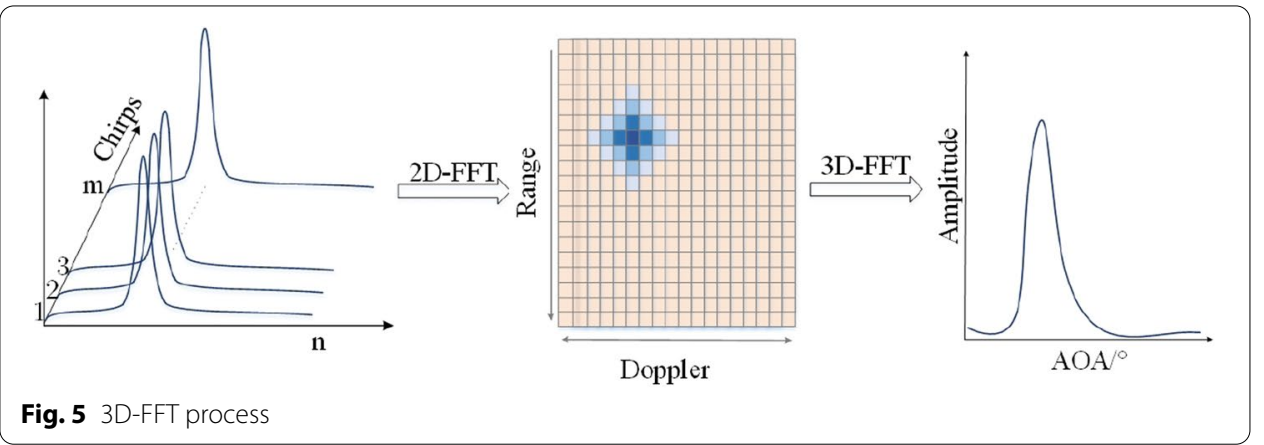

different angles has its own unique law of phase change. As a result, it is possible to separate targets with different frequencies by performing Fourier analysis on multiple echo signals. We propose a 3D-FFT estimation approach and construct RAM to separate the multiple targets. The detailed operation procedure of 3D-FFT is shown as in Fig. 5.

The 3D-FFT is performed for each pair of TX-RX antennas. First, the first FFT (RangeFFT) is performed for each chirp fast sampling point, and its output is stored in the matrix as a continuous row. Then, a second FFT (Doppler-FFT) is performed between the chirps (columns in the matrix). According to the principle of angle estimation, the different wave range differences from the object to each antenna cause a phase change in the 2D-FFT peak, and this phase change can be used to estimate the angle of arrival. Therefore, a third FFT (Angle-FFT) is performed for the phase sequence corresponding to the 2D-FFT peak.

$$
\theta=\sin ^{-1}\left(\frac{\lambda \Delta \phi}{2 \pi d_{i}}\right)
$$

The 3D-FFT can effectively estimate the angle of the target and construct the RAM to achieve multi-target separation. However, it is found that the angle estimated by 3D-FFT is not exactly the same as the actual angle when multi-target vital sign detection is performed in the actual process. Because in reality the human body is not a point target but 
has a certain width, and the angle estimation is not an angle peak but an angle interval. In reality, the human target is not a point target, but it has a certain width. Therefore, the angle estimate of the target is not a peak, but an angle interval.

\subsection{LCMV-ADBF-based multi-human target separation}

ADBF is a beamforming technique that shifts RF complex weighting to the digital baseband, which directly performs weighting operations on digital signals. The weight can be updated according to the sampled data using a certain adaptive algorithm. This results in a narrow main beam direction map, which adaptively enhances the useful signal. At the same time, null steering is formed in the interference direction to suppress unwanted interference and extract useful signal features and information [26]. The ADBF method is applied in the RAM map to improve the angular estimation accuracy and to separate the multiple vital signs. Digital beamforming is achieved digitally by using phase shift, scaling and summation, which in turn can be defined as spatial filtering of the received signal [27]. In this paper, ADBF combined with MIMO extension technology are used at the antenna receiving end to suppress strong interference and directional interference, and effectively extract the vital signs of multiple human.

Each receiving antenna has its own processing chain, and Fig. 6 shows the LCMVADBF processing of the FMCW radar system. For the selection of the adaptive method, the LCMV adaptive method is chosen for the weight update in this paper. In Fig. 6, the signals received by the linear antenna array are used to generate the adaptive weights for each channel by the LCMV method, while the weighted accumulation is used to complete the spatial filtering of the signals.

Beamforming weights $w$ are calculated for each identified angular position $k$ and applied to the ADC sampling data to obtain the output signal $y_{k}(m, n)$.
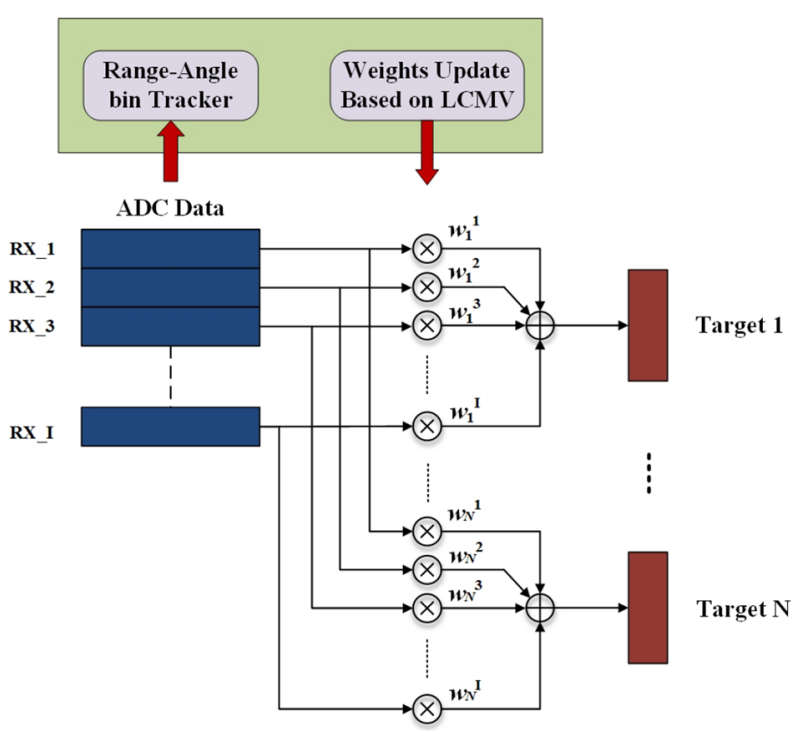

Fig. 6 LCMV-ADBF processing of FMCW radar system 


$$
y_{k}(m, n)=\sum_{i=1}^{I_{R x}} S_{\mathrm{IF}} w_{k}^{i},
$$

where $I_{R x}$ is the number of receiving antenna channels. In time $t$, the simplified expression is

$$
y(t)=w^{H} S(t) .
$$

The LCMV principle is used to ensure that the desired signal direction gain minimizes the total output power.

$$
\left\{\begin{array}{l}
\min P=E\left\{|y(t)|^{2}\right\}=w^{H} R_{S S} w \\
\text { s.t. } w^{H} C=F
\end{array},\right.
$$

where $C$ is the constraint matrix, $F$ is the constrained response vector, and $R_{S S}$ is the sampling covariance matrix. According to the beamforming requirements, the maximum gain constraint is usually in the desired signal direction, while the zero constraint is set in the other interference directions. Then, it is obtained that

$$
\begin{aligned}
& C=\left[1, e^{j \frac{2 \pi}{\lambda} d \sin \left(\theta_{l}\right)}, \ldots, e^{j\left[\left(I_{R x}-1\right) \frac{2 \pi}{\lambda} d \sin \left(\theta_{l}\right)\right]}\right], \\
& F=\left[\begin{array}{llll}
1 & 0 & \cdots & 0
\end{array}\right]^{T} .
\end{aligned}
$$

In practical applications, the Optimal estimation $\hat{R}_{S S}$ is composed of $n$ sampling signal vectors $R_{S S}$.

$$
\hat{R}_{S S}=\frac{1}{n} \sum_{t=1}^{n} S(t) S^{H}(t)
$$

Finally, the optimal weight vector $w$ is calculated as

$$
w=\frac{\hat{R}^{-1} C}{C^{H} \hat{R}^{-1} C} F
$$

The digital adaptive beamforming technique is applied to the RAM constructed by 3D-FFT. It can effectively decrease the negative impact in the direction of interference and mutual interference between multiple human targets, resulting in the effective separation of multiple human targets. Finally, the presence of targets is determined in the distance-angle plane using the target detection CFAR algorithm [28]. The parameters used in this paper are two-dimensional matrix, and the target signal can be extracted by traditional CFAR algorithm. We calculate the energy threshold of the matrix and filter out the clutter below the threshold, so as to retain all target signals. The general processing of CFAR is shown in Fig. 7.

\subsection{Vital signs separation and reconstruction}

For each detected object in the RAM, we extract the phase at a specific distance-angle range. First, we need to apply a circular dynamic tracking algorithm [29] to solve the DC offset problem that occurs during the phase acquisition. At the same time, the negative 


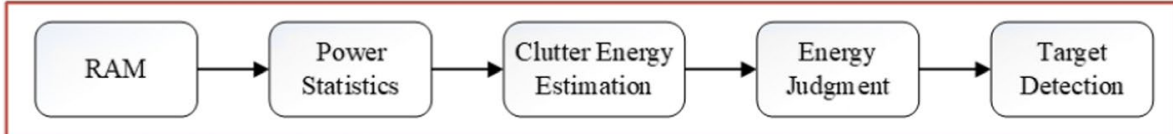

Fig. 7 CFAR processing

effect caused by the low amplitude motion of the human target and the limited value domain problem of the inverse tangent demodulation method itself. We use an extended differential cross-multiply (DACM) algorithm to solve the phase ambiguity [30] and extract the phase signal of the target efficiently. After that, we use an inverse tangent demodulation technique to obtain the phase information of the target. Then, the CSOMP and RA-DWT algorithms [31] are applied to separate and reconstruct our desired respiration and heartbeat signals. Finally, we select data over a period of time for the calculation of respiration and heart rates by the peak detection algorithm [32]. In the range of sampling rates and frequencies, we calculate the inter-peak distance of the signal. We denote the minimum inter-peak distance by $P_{\min }$, and the maximum inter-peak distance by $P_{\max }$. The first peak in the waveform is selected as the valid peak, and the next valid peak is selected such that the distance between the current peak and the previous valid peak is within the interval $\left[P_{\min }, P_{\max }\right]$. In addition, if the amplitude of the signal peak is too high or too low, it can be treated as an invalid peak. After separating the valid peaks, the signal period is based on the average of the inter-peak distances of all valid peaks, and the frequency is regarded as the reciprocal of the period.

\section{Experimental results and analysis}

The experimental platform used in this paper is the AWR1642 radar and DCA1000 data acquisition card, as shown in Fig. 8. The time-division-multiplexing multiple-input-multiple-output (TDM-MIMO) mode of the radar is used, and the two transmitting antennas send FM pulses alternately according to the time gap and obtain the vital signs data of the human targets. In the experiment, the human targets are sitting in front of the radar in a relatively stable posture to verify the effectiveness of the proposed method. Table 1 shows the specification of the radar parameters for multi-target vital signs detection.

\subsection{Vital signs detection of two human targets}

Firstly, we investigate the case of two human targets sitting in the same radial range and the experimental scene in a real environment is shown in Fig. 9a. We use a window of $\mathrm{T} \approx 30 \mathrm{~s}$ to estimate the vital signs. The corresponding schematic diagram of Fig. $9 \mathrm{a}$ is shown in Fig. 9b. The range-angle results of 3D-FFT are shown in Fig. 9c. We find that the 3D-FFT method cannot separate the two human targets at the same distance. This is mainly due to the swaying of the right arm of target 2 that causes a large interference for target extraction. The interference between the targets is suppressed using the proposed LCWV-ADBF method, and the obtained beam direction is shown in Fig. 9d. A gap can be seen at the $0^{\circ}$, and this trap makes the interference power between the two targets reduced, successfully suppressing the undesired signal. The range-angle information of LCMV-ADBF is shown in Fig. 9e. The position information of the two subjects can be 
Table 1 Parameters setting for multi-human target vital sign detection

\begin{tabular}{ll}
\hline Parameters & Specifications \\
\hline Start frequency & $77 \mathrm{GHz}$ \\
Sample rate & $2 \mathrm{Msps}$ \\
Chirp duration & $50 \mathrm{us}$ \\
Sampling points & 100 \\
Range resolution & $4.3 \mathrm{~cm}$ \\
Bandwidth & $4 \mathrm{GHz}$ \\
Angular resolution & $17.5^{\circ}$ \\
Transmitting power & $12.5 \mathrm{dBm}$ \\
\hline
\end{tabular}

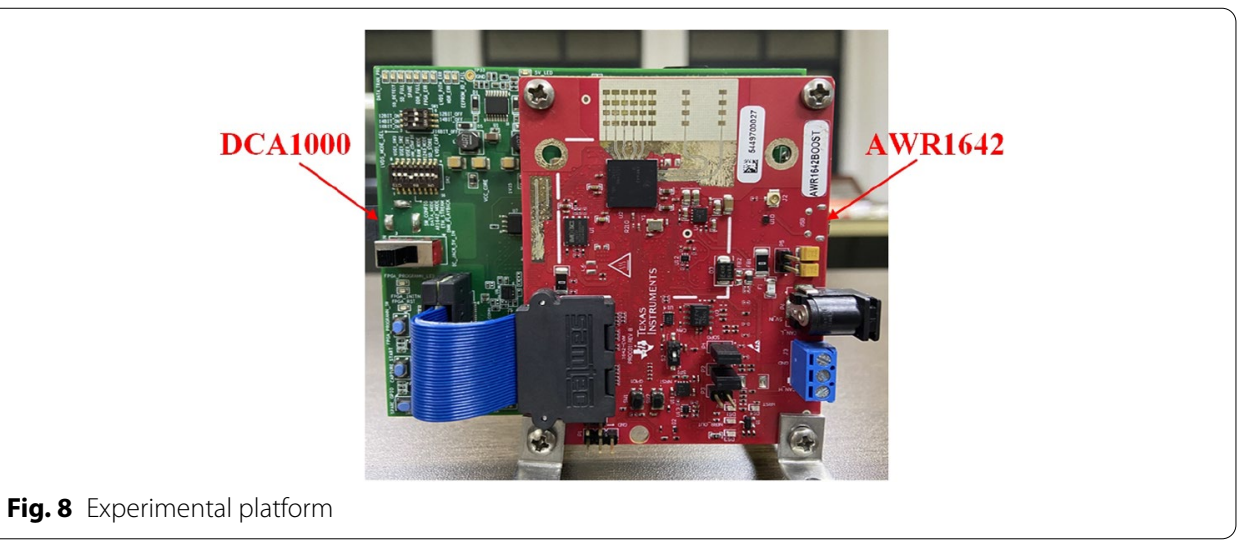

clearly determined, and the two human target signals are successfully separated. The CFAR technique can be used to determine the target location adaptively and filter out static target interference, and the results are given in Fig. 9e.

Before the estimation of respiration and heartbeat rates, we need to solve the problem of DC offset. We take the real part of the radar data as the horizontal axis and the imaginary part as the vertical axis. The results obtained by applying the circle center tracking algorithm to DC offset correction are shown in Fig. 10. The green point is the experimental data, and the purple point is the data after DC offset correction. We can clearly see that the center of the green circle is offset from the center of the purple circle.

After separating the human targets, the CS-OMP algorithm is applied to detect the vital signs of the two human targets, and the vital signs signals are given in Fig. 11. Figure 11a, c shows the time domain waveform of the respiratory and heartbeat signals of target 1 and 2, respectively, which are presented as quasi-periodic. Figure 11b, d shows the frequency domain waveforms of respiratory and heartbeat signals of target 1 and 2, respectively. The respiratory rates of target 1 and 2 are $0.72 \mathrm{~Hz}$ and $0.56 \mathrm{~Hz}$, respectively. The heartbeat rates are $1.34 \mathrm{~Hz}$ and $1.20 \mathrm{~Hz}$, respectively, and the results are in accordance with the human respiratory and heartbeat frequencies.

\subsection{The level of agreement for respiration and heartbeat rates}

Moreover, a special experimental requirement is imposed on the two subjects in order to validate the effectiveness of the method proposed. The subjects 1 and 2 are made to 


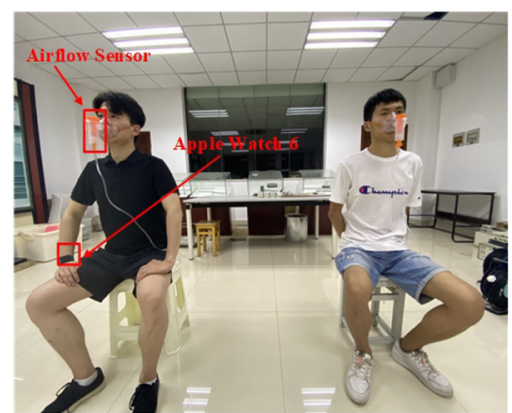

(a) Experimental scene.

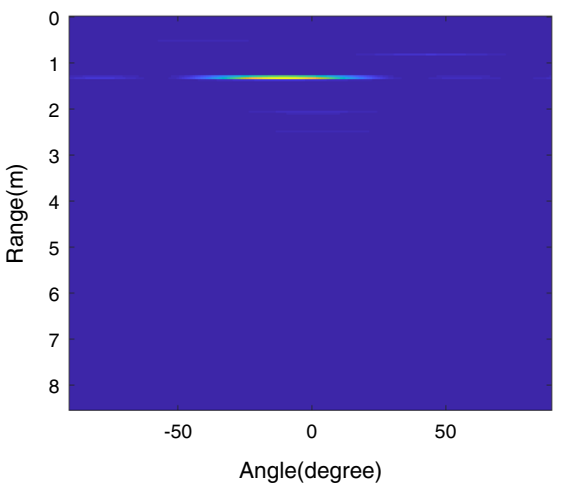

(c) Range-angle map after 3D-FFT.

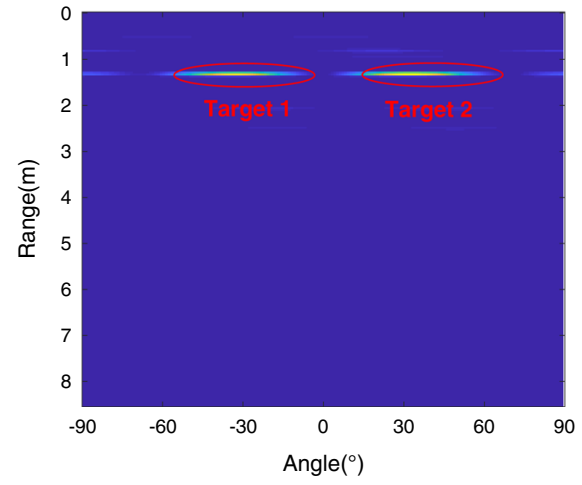

(e) Range-angle map after ADBF.

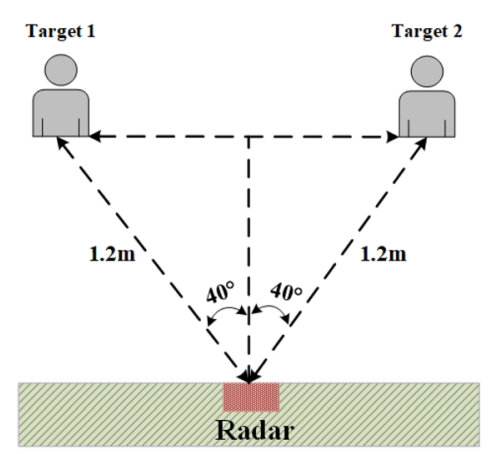

(b) Schematic.

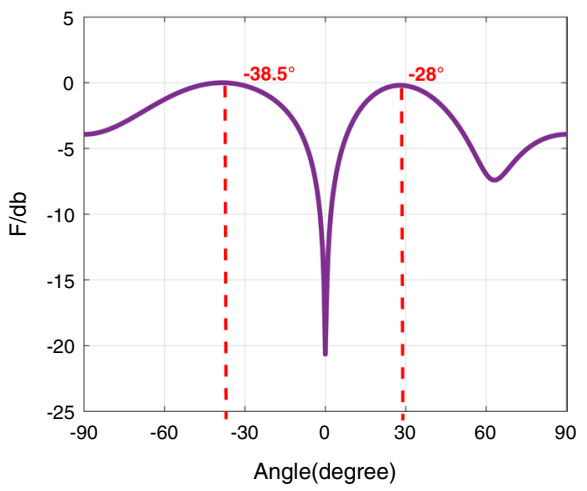

(d) Beam direction map.

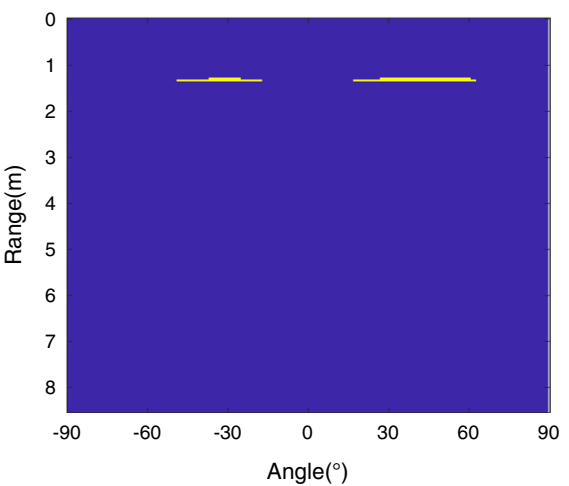

(f) Target extraction after CFAR.

Fig. 9 Two human targets detection

alternate between normal breathing and hold breathing, and each breathing pattern is continued for $10 \mathrm{~s}$. The phase unfolding signals of the vital characteristics of the two individuals are obtained in Fig. 12. During breathing, the small-amplitude heartbeat waveform is attached to the respiratory waveform. When the tester holds breath, only the small-amplitude heartbeat waveform is presented. This proves the effectiveness of the proposed method for two target vital signs detections. During breathing, a smallamplitude heartbeat waveform is attached to the respiratory waveform. When breathing is held, small-amplitude heartbeat waveforms are presented. Moreover, it is also 


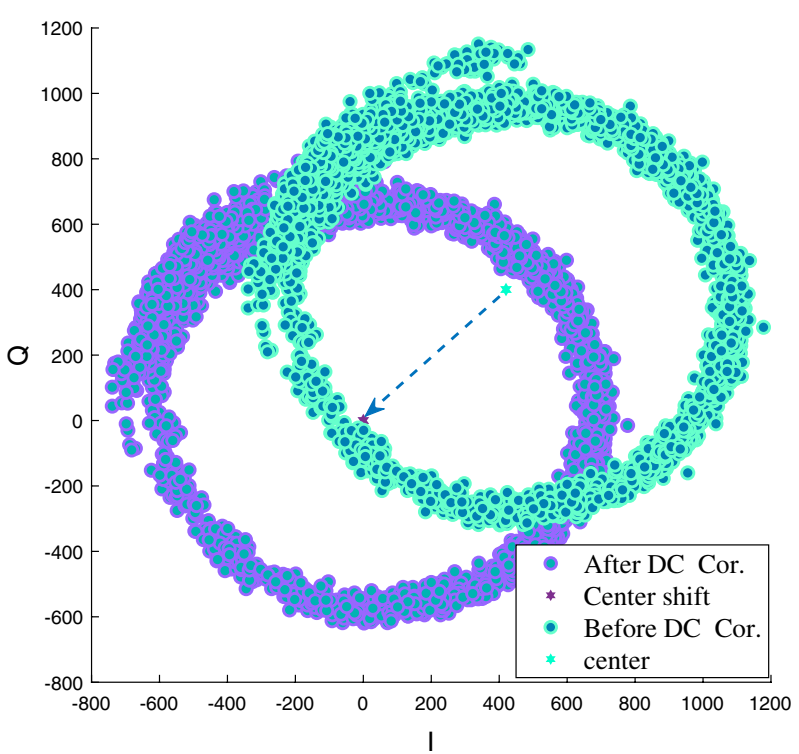

Fig. 10 DC offset correction

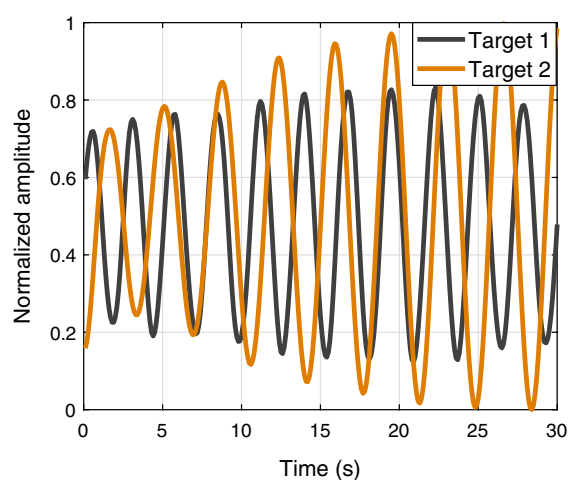

(a) Time-domain waveform of respiratory signal.

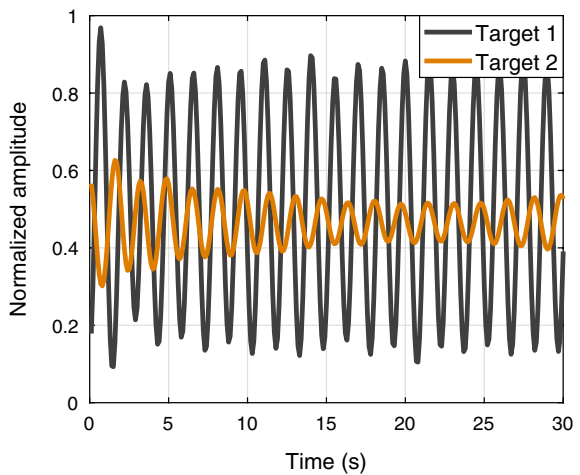

(c) Time-domain waveform of heartbeat signal.

Fig. 11 Time and spectrum of two human targets

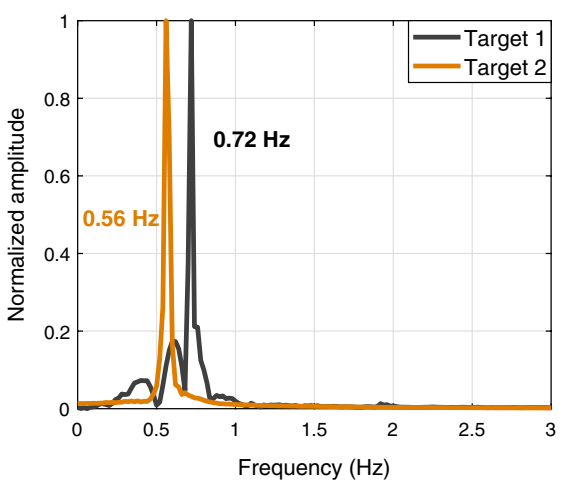

(b) Specturm of respiratory signal.

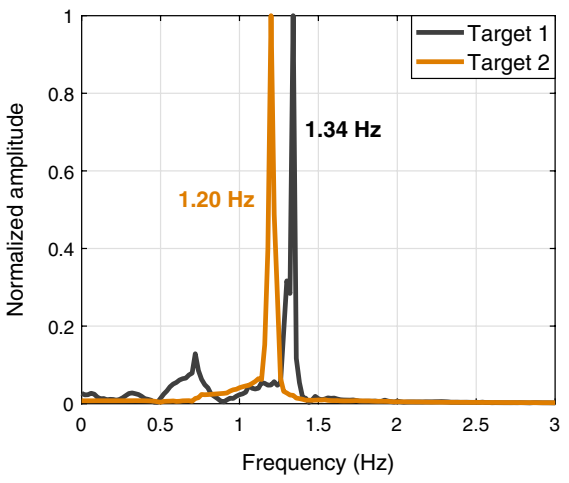

(d) Specturm of heartbeat signal. 


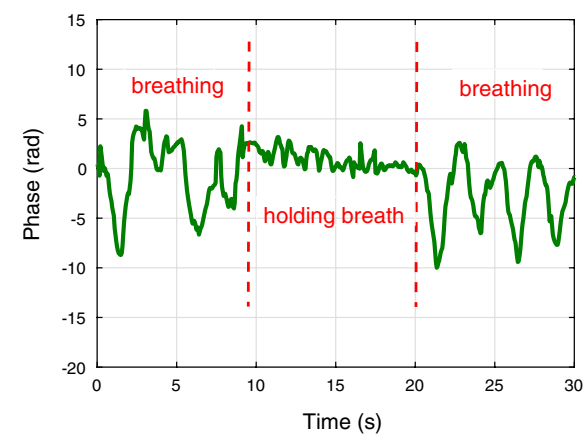

(a) Subject 1 for vital signal after phase unwrapping.

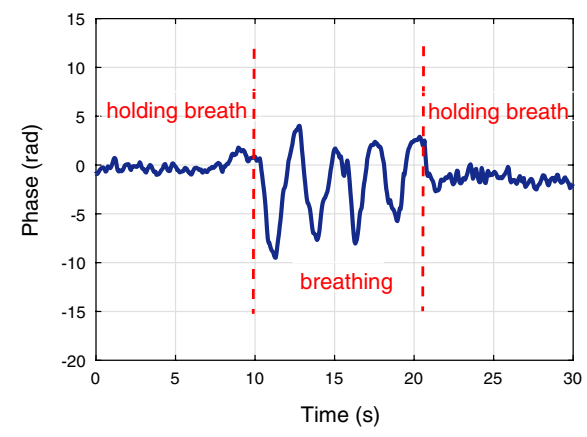

(b) Subject 2 for vital signal after phase unwrapping.

Fig. 12 Two human targets with alternate breathing

observed from the figure that the two human targets complete the respiratory pattern shift at almost the same moment, which validate the effectiveness of the proposed method.

\subsection{Vital sign detection for three human targets}

In order to verify the scalability of the system, experiments are carried out considering three human targets, and the scenario in real environment is given in Fig. 13a. Similar to Figs. 9, 13c shows the experimental results obtained by using the proposed method at different angles for three subjects at a distance of $1 \mathrm{~m}$. The use of LCMV-ADBF technology can not only suppress the influence of the interference on angle of arrival (AOA) direction, but also reduce the mutual influence among multiple targets. As shown in Fig. 13c, without LCMV-ADBF, the SNR of human target 2 is greater than that of human targets 1 and 3, resulting in the inability to distinguish multiple targets in the RAM. As in Fig. 13d, the ADBF is used to generate trapped waves around $0^{\circ}$ of human target 2 to reduce the effect of human target 2 on the remaining human targets. Figure 13e shows that when combines ADBF with CFAR, multiple targets can be clearly distinguished in RAM. It should be noted that since the human targets occupy a certain angle, only three human targets can be occupied in the radar field of view, we will verify more human targets in the future.

In the experiment, eight males and seven females are invited to perform multi-target vital signs of radar data acquisition in two-person and three-person groups, respectively. The length of 1-min radar data is intercepted, and the proposed LCMV-ADBF algorithm is applied to the separated the human targets to separate and reconstruct using the wavelet transform and CS-OMP method. The peak detection method was used for respiration and heartbeat rates estimation, and the Pearson coefficient [33] is used to calculate the degree of agreement between the results obtained by proposed method and the reference sensors, and the results are shown in Tables 2 and 3.

From the experimental results, it can be seen that in the case of two human targets, the level of agreement of the respiratory obtained by CS-OMP and RA-DWT methods and the contact devices are $89 \%$ and $90 \%$, respectively, and the corresponding degree of agreement of heartbeat are $87 \%$. In the case of three human targets, the degree of agreement of the respiratory obtained by two methods and the contact 


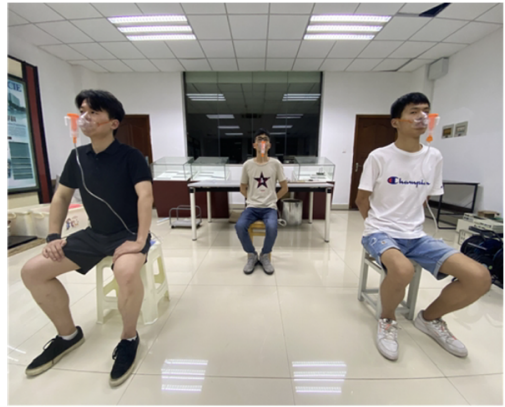

(a) Experimental scene.

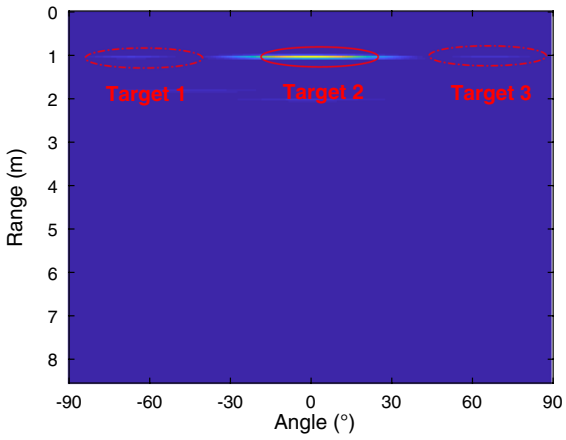

(c) Range-angle map after 3D-FFT.

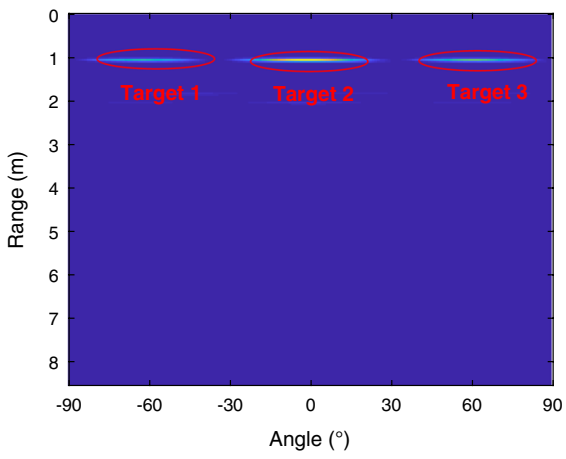

(e) Range-angle map after ADBF.

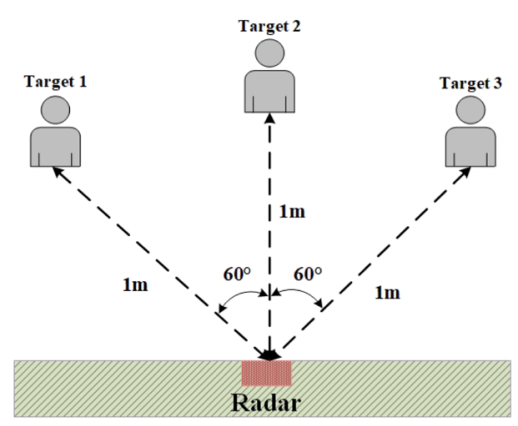

(b) Schematic.

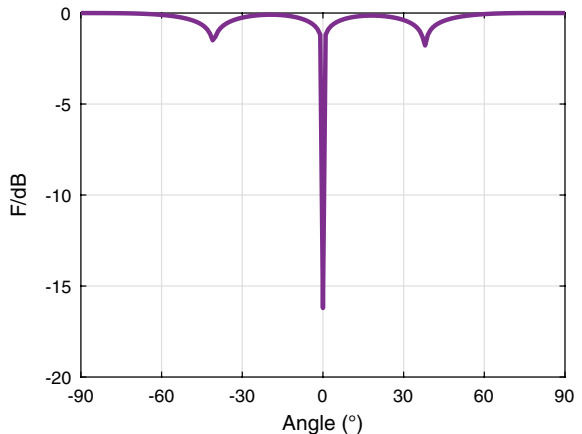

(d) Beam direction map.

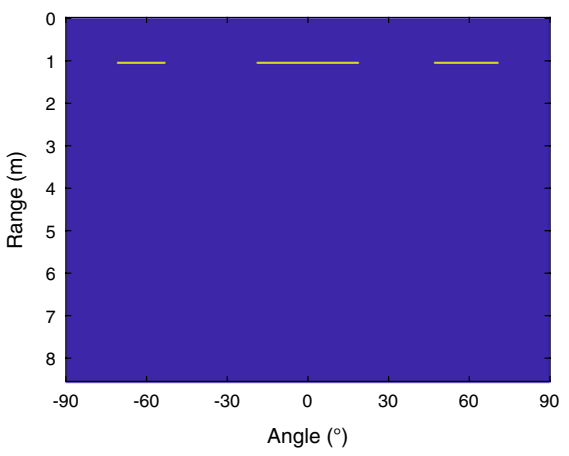

(f) Target extraction after CFAR.

Fig. 13 Three-human target detection

devices are both $87 \%$, respectively, and the degree of agreement heartbeat are both $87 \%$. The accuracy of respiratory and heartbeat rates will be further reduced. Compared with [31], the single human respiration and heart rates obtained by the same algorithm are $96 \%$ and $94 \%$, respectively. It is obvious that there is a small decrease in the accuracy of detection of multiple human target vital signals. It is possible because by increasing the number of human targets, the motion artifacts such as hand gestures or small movements like shaking of legs will introduce interference. Moreover, when the human bodies are too close together, the signal overlap will be caused between multiple targets, and the human bodies with strong vital signals will cover up the weak vital signals between neighboring human target, which in turn 
Table 2 Comparison of the respiration and heartbeat rates between two targets

\begin{tabular}{|c|c|c|c|c|c|}
\hline \multirow[t]{2}{*}{ Category } & \multirow[t]{2}{*}{ Groups } & \multirow[t]{2}{*}{ Targets } & \multirow[t]{2}{*}{ Airflow sensor } & \multicolumn{2}{|c|}{ AWR1642 radar sensor } \\
\hline & & & & CS-OMP & RA-DWT \\
\hline \multirow[t]{7}{*}{ Respiration } & 1 & Male 1 & 21 & 18 & 21 \\
\hline & & Male 2 & 23 & 21 & 22 \\
\hline & 2 & Female 1 & 24 & 26 & 26 \\
\hline & & Female 2 & 26 & 25 & 28 \\
\hline & 3 & Male 3 & 20 & 18 & 22 \\
\hline & & Female 3 & 24 & 25 & 25 \\
\hline & PCC & - & - & $89 \%$ & $90 \%$ \\
\hline \multirow[t]{7}{*}{ Heartbeat } & 1 & Male 1 & 70 & 75 & 68 \\
\hline & & Male 2 & 77 & 74 & 79 \\
\hline & 2 & Female 1 & 63 & 66 & 67 \\
\hline & & Female 2 & 84 & 80 & 80 \\
\hline & 3 & Male 3 & 71 & 76 & 76 \\
\hline & & Female 3 & 68 & 71 & 72 \\
\hline & $P C C$ & - & - & $87 \%$ & $87 \%$ \\
\hline
\end{tabular}

Table 3 Comparison of the respiration and heartbeat rates between three targets

\begin{tabular}{|c|c|c|c|c|c|}
\hline \multirow[t]{2}{*}{ Category } & \multirow[t]{2}{*}{ Groups } & \multirow[t]{2}{*}{ Targets } & \multirow[t]{2}{*}{ Airflow sensor } & \multicolumn{2}{|c|}{ AWR1642 radar sensor } \\
\hline & & & & CS-OMP & RA-DWT \\
\hline \multirow[t]{10}{*}{ Respiration } & \multirow[t]{3}{*}{4} & Male 4 & 21 & 20 & 20 \\
\hline & & Male 5 & 22 & 24 & 21 \\
\hline & & Male 6 & 27 & 25 & 24 \\
\hline & \multirow[t]{3}{*}{5} & Female 4 & 19 & 18 & 19 \\
\hline & & Female 5 & 24 & 22 & 21 \\
\hline & & Female 6 & 21 & 23 & 17 \\
\hline & \multirow[t]{3}{*}{6} & Male7 & 18 & 16 & 19 \\
\hline & & Male8 & 25 & 23 & 22 \\
\hline & & Female7 & 27 & 29 & 24 \\
\hline & PCC & - & - & $87 \%$ & $87 \%$ \\
\hline \multirow[t]{10}{*}{ Heartbeat } & \multirow[t]{3}{*}{4} & Male 4 & 68 & 65 & 64 \\
\hline & & Male 5 & 88 & 82 & 84 \\
\hline & & Male 6 & 73 & 69 & 76 \\
\hline & \multirow[t]{3}{*}{5} & Female 4 & 72 & 68 & 75 \\
\hline & & Female 5 & 70 & 74 & 66 \\
\hline & & Female 6 & 85 & 79 & 80 \\
\hline & \multirow[t]{3}{*}{6} & Male7 & 70 & 73 & 76 \\
\hline & & Male8 & 85 & 81 & 81 \\
\hline & & Female7 & 71 & 75 & 67 \\
\hline & $P C C$ & - & - & $85 \%$ & $85 \%$ \\
\hline
\end{tabular}

affects the detection matching rate. Nevertheless, the above experiments show our contributions in the field of multi-target vital signs extraction and prove the feasibility of multi-target vital signs detection. 


\title{
5 Conclusion
}

In this paper, we estimated the range and angle of multiple targets using 3D-FFTbased method. Then, the LCMV-ADBF technique was proposed for interference suppression to enhance the desired respiratory and heartbeat signal. The CS-OMP and RA-DWT methods were applied to separate and construct the respiration and heartbeat signals of the separated human target. The experimental results were compared with the reference ones of contact sensors (Airflow sensors and Apple Watch 6). The results showed that the proposed method could effectively extract the respiration and heartbeat signals from multiple human targets. Compared with the contacted sensors, the degree of agreement for respiration and heartbeat rates are more than $87 \%$ for two human targets and $85 \%$ for three human targets. In the following study, we will make more efforts on the detection of vital signals under target movement.

\section{Abbreviations}

FMCW: Frequency-modulated continuous wave; 3D-FFT: Three dimensions fast Fourier transform; LCMV-ADBF: Linear constrained minimum variance-based adaptive beamforming; CS-OMP: Compressive sensing based on orthogonal matching pursuit; RA-DWT: Rigrsure adaptive soft threshold noise reduction based on discrete wavelet transform; MUSIC: Multiple signal classification; SNR: Signal-to-noise ratio; MS: Mechanical scanning; RF: Radio frequency; IF: Intermediate frequency; CFAR: Constant-false-alarm-rate; TDM: Time-division multiplexing; MIMO: Multiple-input-multiple-output; RAM: Range-angle map; AOA: Angle of arrival.

\section{Acknowledgements}

This work was supported by the School of Communication and Information Engineering, Chongqing University of Posts and Telecommunications.

\section{Authors' contributions}

The research and results of this specific publication are a long-term collaboration between the authors based on MIMO radar applications. For the present manuscript, we achieve vital signs detection of multiple human targets based on MIMO radar using adaptive beamforming techniques. All authors read and approved the final manuscript.

\begin{abstract}
Author Information
Yong Wang (Member, IEEE) received the B.S., M.S. and Ph.D. degrees from Harbin Institute of Technology, Harbin, China, in 2010, 2012 and 2018, respectively. He is currently a Lecturer with Chongqing University of Posts and Telecommunications, Chongqing, China. From January 2014 to June 2015, he was a visiting Ph.D. student at the Department of Electrical and Computer Engineering, University of Toronto, Canada. His research interests include millimeter sensing, humancomputer interaction $(\mathrm{HCl})$, deep learning and resource management.

Yuzhu Shui was born in Jiangsu, China, in 1996. He received his B.S. degree from Jiangsu Ocean University in 2019, and he currently pursuing his master degree at Chongqing University of Posts and Telecommunications, Chongqing, China. Her research interests include millimeter-wave radar and vital signs detection.

Xiaobo Yang received the B.S. degree from the Chengdu Institute of Meteorology, Chengdu, China, in 2000, and the M.S. degree from the Chongqing University of Posts and Telecom-munications, Chongqing, China, in 2008. From January 2016 to July 2016, she was a Visiting Scholar with the University of California at San Diego, San Diego, USA. She is currently a Lec-turer with the Chongqing University of Posts and Telecommunications. Her research interests include indoor localization, machine learning, and deep neural networks.

Zhaoyu Li received the B.S. degree from the Chongqing University of Posts and Telecommunications, Chongqing, China (Associate Professor, Master Supervisor). She was engaged in 1 provincial and ministerial projects. She has published 7 research papers and 2 authorized patents. She is engaged in the research and teaching of mobile communication technology.

Wen Wang received the B.S. degree from Nanchang Hangkong University, Jiangxi, China, in 2018. He received his Masters degree from Chongqing University of Posts and Telecommunication in 2021. His research interests include deep learning
\end{abstract} and radar signal processing.

\section{Funding}

This work was supported in part by the National Natural Science Foundation of China under Grant 61901076 and Grant 61771083; in part by the National Science Foundation of Chongqing under Grant cstc2020jcyj-msxmX0865; in part by the China Postdoctoral Science Foundation under Grant 2021M693773; and in part by the Science and Technology Research Program of Chongqing Education Commission under Grant KJQN201900603, Grant KJZD-K202000605, and Grant KJQN202000630

\section{Availability of data and materials}

All data generated or analyzed during this study are included in this paper. 


\section{Declarations}

Ethics approval and consent to participate

All procedures performed in this paper were in accordance with the ethical standards of research community.

\section{Consent for publication}

Not applicable.

\section{Competing interests}

The authors declare that they have no competing interests.

Received: 8 July 2021 Accepted: 11 October 2021

Published online: 24 October 2021

\section{References}

1. S. Sidney, W.D. Rosamond, V.J. Howard, R.V. Luepker, The heart disease and stroke statistics-2013 update and the need for a national cardiovascular surveillance system. Circulation 127(1), 21-23 (2012)

2. S.N. Bose, A. Verigan, J. Hanson, L. Ahumada, S. Ghazarian, N. Goldenberg, A. Stock, J. Jacobs, Early identification of impending cardiac arrest in neonates and infants in the cardiovascular ICU: a statistical modelling approach using physiologic monitoring data. Cardiol. Young 29(1), 1340-1348 (2019)

3. N. Bayasi, T. Tekeste, H. Saleh, B. Mohammad, A. Khandoker, M. Ismail, Low-power ecg-based processor for predicting ventricular arrhythmia. IEEE Trans. Very Large Scale Integr. Syst. 24(5), 1962-1974 (2016)

4. C. Massaroni, A. Nicolò, D. Lo Presti, M. Sacchetti, S. Silvestri, E. Schena, Contact-based methods for measuring respiratory rate. Sensors $\mathbf{1 9}(4), 908$ (2019)

5. S. Kano, Y. Dobashi, M. Fujii, Silica nanoparticle-based portable respiration sensor for analysis of respiration rate, pattern, and phase during exercise. IEEE Sens. Lett. 2(1), 1-4 (2018)

6. S. Milici, A. Lázaro, R. Villarino, D. Girbau, M. Magnarosa, Wireless wearable magnetometer-based sensor for sleep quality monitoring. IEEE Sens. J. 18(5), 2145-2152 (2018)

7. K. Uemura, T. Kawada, M. Sugimachi, A novel minimally occlusive cuff method utilizing ultrasound vascular imaging for stress-free blood pressure measurement: a-proof-of-concept study. IEEE Trans. Biomed. Eng. 66(4), 934-945 (2019)

8. Y. Xu, S. Dai, S. Wu, J. Chen, G. Fang, Vital sign detection method based on multiple higher order cumulant for ultrawideband radar. IEEE Trans. Geosci. Remote Sens. 50(4), 1254-1265 (2012)

9. Z. Peng, J.M. Muñoz-Ferreras, Y. Tang, C. Liu, R. Gómez-García, L. Ran, C. Li, A portable fmcw interferometry radar with programmable low-if architecture for localization, isar imaging, and vital sign tracking. IEEE Trans. Microw. Theory Tech. 65(4), 1334-1344 (2017)

10. M. Nosrati, S. Shahsavari, S. Lee, H. Wang, N. Tavassolian, A concurrent dual-beam phased-array doppler radar using MIMO beamforming techniques for short-range vital-signs monitoring. IEEE Trans. Antennas Propag. 67(4), 2390-2404 (2019)

11. J. Lee, J. Park, Y. Kim, M. Woo, Affective change with variations in zumba fitness intensity as measured by a smartwatch. Percept. Motor Skills 128(1), 2255-2278 (2021)

12. H. Hong, L. Zhang, H. Zhao, H. Chu, C. Gu, M. Brown, X. Zhu, C. Li, Microwave sensing and sleep: noncontact sleepmonitoring technology with microwave biomedical radar. IEEE Microw. Mag. 20(8), 18-29 (2019)

13. C.B. Pereira, X. Yu, T. Goos, I. Reiss, T. Orlikowsky, K. Heimann, B. Venema, V. Blazek, S. Leonhardt, D. Teichmann, Noncontact monitoring of respiratory rate in newborn infants using thermal imaging. IEEE Trans. Biomed. Eng. 66(4), 1105-1114 (2019)

14. A. Singh, S. Rehman, S. Yongchareon, P. Chong, Multi-resident non-contact vital sign monitoring using radar: a review. IEEE Sens. J. 22(1), 4061-4084 (2021)

15. A. Ahmad, J.C. Roh, D. Wang, A. Dubey, in Proceedings of the 2018 IEEE Radar Conference (RadarConf18), Vital Signs Monitoring of Multiple People Using a fmcw Millimeter-Wave Sensor (IEEE, Oklahoma City, 2018), pp. 1450-1455

16. G.-W. Fang, C.-Y. Huang, C.-L. Yang, in Proceedings of the 2019 IEEE MTT-S International Microwave Biomedical Conference (IMBioC), Simultaneous Detection of Multi-target Vital Signs Using eemd Algorithm Based on fmcw Radar (IEEE, Nanjing, 2019), pp. 1-4

17. G. Wang, C. Gu, T. Inoue, C. Li, A hybrid fmcw-interferometry radar for indoor precise positioning and versatile life activity monitoring. IEEE Trans. Microw. Theory Tech. 62(11), 2812-2822 (2014)

18. H. Lee, B.-H. Kim, J.-K. Park, J.-G. Yook, A novel vital-sign sensing algorithm for multiple subjects based on 24-ghz fmcw doppler radar. Remote Sens. 11(10), 1237 (2019)

19. K. Wang, J.-F. Gu, F. Ren, K. Wu, A multitarget active backscattering 2-d positioning system with superresolution time series post-processing technique. IEEE Trans. Microw. Theory Tech. 65(5), 1751-1766 (2017)

20. A.B. Baral, M. Torlak, Impact of number of noise eigenvectors used on the resolution probability of music. IEEE Access 7, 20023-20039 (2019)

21. S. Kim, K.-K. Lee, Low-complexity joint extrapolation-music-based 2-d parameter estimator for vital fmcw radar. IEEE Sens. J. 19(6), 2205-2216 (2019)

22. S.M.M. Islam, E. Yavari, A. Rahman, V.M. Lubecke, O. Boric-Lubecke, in Proceedings of the 2019 IEEE Radio and Wireless Symposium (RWS), Multiple Subject Respiratory Pattern Recognition and Estimation of Direction of Arrival Using Phasecomparison Monopulse Radar (IEEE, Orlando, 2019), pp. 1-4

23. S.M.M. Islam, O. Boric-Lubecke, V.M. Lubekce, Concurrent respiration monitoring of multiple subjects by phase-comparison monopulse radar using independent component analysis (ICA) with jade algorithm and direction of arrival (DOA). IEEE Access 8, 73558-73569 (2020) 
24. G.-W. Fang, C.-Y. Huang, C.-L. Yang, Switch-based low intermediate frequency system of a vital sign radar for simultaneous multitarget and multidirectional detection. IEEE J. Electromagn. RF Microw. Med. Biol. 4(4), 265-272 (2020)

25. J. Xiong, H. Zhang, H. Hong, H. Zhao, X. Zhu, C. Li, in 2020 IEEE Radio and Wireless Symposium (RWS), Multi-Target Vital Signs Detection Using Simo Continuous-Wave Radar with Dbf Technique (San Antonio, TX, USA, IEEE, 2020), pp. 194-196

26. G. Pei, L. Ming, L. Xiang, An adaptive beamforming method at subarray level for planar array with low sidelobes. Radar Sci. Technol. 10, 519-523 (2012)

27. M. Rameez, M. Dahl, M.I. Pettersson, in 2018 IEEE Radar Conference (RadarConf18), Adaptive Digital Beamforming for Interference Suppression in Automotive Fmcw Radars (IEEE, Oklahoma City, 2018), pp. 252-256

28. Q. Zheng, L. Yang, Y. Xie, J. Li, T. Hu, J. Zhu, C. Song, Z. Xu, A target detection scheme with decreased complexity and enhanced performance for range-doppler fmcw radar. IEEE Trans. Instrum. Meas. 70, 1-13 (2020)

29. M.-C. Huang, J.J. Liu, W. Xu, C. Gu, C. Li, M. Sarrafzadeh, A self-calibrating radar sensor system for measuring vital signs. IEEE Trans. Biomed. Circuits Syst. 10(2), 352-363 (2016)

30. S. Dong, Y. Zhang, C. Ma, C. Zhu, Z. Gu, Q. Lv, B. Zhang, C. Li, L. Ran, Doppler cardiogram: a remote detection of human heart activities. IEEE Trans. Microw. Theory Tech. 68(3), 1132-1141 (2020)

31. Y. Wang, W. Wang, M. Zhou, A. Ren, Z. Tian, Remote monitoring of human vital signs based on 77-ghz mm-wave fmcw radar. Sensors 20(10), 2999 (2020)

32. X. Yang, G. Sun, K. Ishibashi, in Proceedings of the 201739 th Annual International Conference of the IEEE Engineering in Medicine and Biology Society (EMBC), Non-contact Acquisition of Respiration and Heart Rates Using Doppler Radar with Time Domain Peak-detection Algorithm(Jeju, Korea, 2017), pp. 2847-2850

33. C. Jung, M. Groth, K.U. Petersen, A. Hammel, F. Brinkert, E. Grabhorn, S.A. Weidemann, J. Busch, G. Adam, J. Herrmann Hepatic shear wave elastography in children under free-breathing and breath-hold conditions. Eur. Radiol. 27(12), 5337-5343 (2017)

\section{Publisher's Note}

Springer Nature remains neutral with regard to jurisdictional claims in published maps and institutional affiliations.

\section{Submit your manuscript to a SpringerOpen ${ }^{\circ}$ journal and benefit from:}

- Convenient online submission

- Rigorous peer review

- Open access: articles freely available online

- High visibility within the field

- Retaining the copyright to your article

Submit your next manuscript at $\mathbf{s p r i n g e r o p e n . c o m ~}$ 